Pacific Journal of Mathematics

INTERSECTIONAL PROPERTIES OF CERTAIN FAMILIES OF

B. E. FULBRIGHT 


\title{
INTERSECTIONAL PROPERTIES OF CERTAIN FAMILIES OF COMPACT CONVEX SETS
}

\author{
B. E. FULlbRight
}

Let $p$ and $q$ be integers with $p \geqq q \geqq 2$. A family $\dddot{\mho}$ of compact convex subsets of a finite dimensional linear space is said to have the $(p, q)$-property if $\widetilde{F}$ contains at least $p$ sets and from each $p$ sets of $\mathfrak{F}$ some $q$ have a common point. In this paper a family $\mathfrak{F}$ is defined to have the $(p, q, k)$-property in a $n$-dimensional normed linear space if $\widetilde{F}$ has the $(p, q)$-property and an additional property which is measured by $k$, with $0 \leqq$ $k \leqq 1$. In some sense $k$ measures the "squareness" of the members of $\mathfrak{F}$. The main result is that if $k>0$, there exists a positive integer $P_{n}(p, q, k)$ such that each family $\widetilde{F}$ with the $(p, q, k)$-property in a $n$-dimensional normed linear space can be partitioned into $P_{n}(p, q, k)$ subfamilies each with a nonempty intersection.

Hadwiger and Debrunner have considered the following question: Is there a positive integer $N(p, q, n)$ such that every finite family $\widetilde{F}$ of sets in $E^{n}$ with the $(p, q)$-property can be partitioned into $N(p, q, n)$ subfamilies each of which has a nonempty intersection?

1. Preliminaries. Let $\mathfrak{F}$ be a family of nonempty subsets of a space $X$ and $r$ a positive integer. The family $\mathfrak{F}$ is said to be $r$-pierceable if there exists a subset $F$ of $X$ consisting of $r$ or fewer points such that $A \cap F \neq \varnothing$ for all $A \in \mathfrak{\mho}$. If $\widetilde{\mho}$ is $r$-pierceable for some $r$, then define $|\mathfrak{F}|=\min \{r: \mathfrak{F}$ is $r$-pierceable $\}$. If $\mathfrak{F}$ is not $r$-pierceable for any positive integer $r$, then define $|\mathfrak{F}|=\infty$.

The following lemma is a generalization of a well-known theorem about the intersection of families of closed and compact subsets of $X$ with the finite intersectional property. The proof is routine and is omitted.

LEMma 1.1. Let $\mathfrak{F}$ be a family of closed and compact subsets of $X$ and $m$ a positive integer. If $|\mathscr{G}| \leqq m$ for each nonempty finite subfamily $\mathscr{G}$ of $\mathfrak{F}$, then $|\mathfrak{F}| \leqq m$.

The symbol $L^{n}$ will denote the $n$-dimensional normed real linear space consisting of all $n$-tuples of real numbers whose norm is given by $\left\|\left(\alpha_{1}, \cdots, \alpha_{n}\right)\right\|=\max \left|\alpha_{i}\right|$, and the symbol $B^{n}$ will denote the closed unit ball of $L^{n}$. To each ordered pair $(x, A)$ where $A$ is a compact subset of $L^{n}$ and $x \in A$, associate real numbers $I(x, A)$ and $E(x, A)$ defined by $I(x, A)=\sup \left\{\lambda: x+\lambda B^{n} \subset A\right\}$ and $E(x, A)=\inf \{\lambda \geqq 0: x+$ $\left.\lambda B^{n} \supset A\right\}$. 
Let $k$ be a real number with $0 \leqq k \leqq 1$. Then a family $\mathfrak{F}$ of nondegenerate compact convex subsets of $L^{n}$ is said to have the $(p, q, k)$-property in $L^{n}$, if $\widetilde{F}$ has the $(p, q)$-property and for each $A \in \widetilde{F}$ there exists a point $x \in A$ such that $(I(x, A) / E(x, A)) \geqq k$. Note that since $A$ is a nondegenerate set $E(x, A) \neq 0$; consequently, the above ratio is defined and satisfies the inequality $0 \leqq(I(x, A) / E(x, A)) \leqq 1$.

For integers $p, q$, and $n$ with $p \geqq q \geqq 2, n \geqq 1$ and real number $k$ with $0 \leqq k \leqq 1$ define $P_{n}(p, q, k)=\sup \{|\mathfrak{F}|: \mathfrak{F}$ has the $(p, q, k)$ property in $\left.L^{n}\right\}$.

It is easy to see that if $\widetilde{F}$ is a family of subsets of $L^{n}$ with the $(p, q)$-property then $|\mathfrak{F}| \leqq P_{n}(p, q, 0)$. This fact together with Lemma 1.1 implies that the number $N(p, q ; n)$ as defined by Hadwiger and Debrunner [2] is the same as $P_{n}(p, q, 0)$. By a slight alteration of the proof of Hadwiger and Debrunner's theorem [2] one can obtain the following theorem.

THEOREM 1.2. If $\mathfrak{F}$ is a finite family of subsets of $L^{n}$ with the $(p+n, q+n-1)$-property, there exists a subfamily $\mathscr{C}$ of $\widetilde{F}$ such that $|\mathfrak{F}| \leqq|\mathscr{C}|+1$ and either $\mathscr{C}$ has the $(p, q)$-property or $|\mathscr{H}| \leqq$ $p-q+1$.

Corollary 1.3. $P_{n}(p+n, q+n-1, k) \leqq P_{n}(p, q, k)+1$ for all $k \in[0,1]$.

Proof. This is a consequence of Theorem 1.2 and Lemma 1.1.

CoROLlaRY 1.4. If $p \geqq q \geqq n+1 \geqq 2$ and $n q \geqq(n-1) p+n+$ 1 , then $P_{n}(p, q, k)=p-q+1$ for all $k \in[0,1]$.

Proof. By means of an example it is not difficult to show that $P_{n}(p, q, k) \geqq p-q+1$. Helly's theorem [4] implies that $P_{n}(p, p, k)=$ 1 for $p \geqq n+1$ and $k \in[0,1]$. The corollary now follows from Corollary 1.3 and by induction on $p-q$.

2. The main result. Let $\mathfrak{F}=\left\{A_{i}: i=1, \cdots\right\}$ where $A_{i}$ is the convex hull of the set $\{(1 / i, 0),(0,1-1 / i)\}$. Then $\widetilde{F}$ has the $(2,2,0)$ property in $L^{2}$ and $|\mathfrak{F}|=\infty$. Consequently, $P_{2}(2,2,0)=\infty$. Similar examples can be constructed in $L^{n}, n \geqq 2$, to show that $P_{n}(n, n, 0)=$ $\infty$. Consequently, $P_{n}(p, q, 0)=\infty$ for all $p \geqq q \geqq 2$ and $q \leqq n$. However, the following theorem implies that the situation is somewhat different for $k>0$. 
THEOREM 2.1. Let $p, q$, and $n$ be positive integers with $p \geqq q \geqq$ 2 and $k$ a real number with $0<k \leqq 1$. Then $P_{n}(p, q, k)$ is finite.

Proof. Let $N_{1}$ be the minimum number of translates of $k B^{n}$ required to cover $(1+k) B^{n}$ and $N_{2}$ the minimum number of translates of $k^{2} B^{n}$ with center of symmetry on $\mathrm{bd}\left[(1+k) B^{n}\right]$ required to cover bd[(1+k)B $B^{n}$. Lemma 1.1 implies that it suffices to show that $|\mathfrak{F}| \leqq(p-q+1)\left(N_{1}+N_{2}\right)$ whenever $\mathfrak{F}$ is a finite family of sets with the $(p, q, k)$-property in $L^{n}$.

Let $\mathfrak{F}=\left\{A_{i}: i=1, \cdots, m\right\}$ have the $(p, q, k)$-property in $L^{n}$. For each $A_{i} \in \widetilde{F}$ there exists a point $x_{i} \in A_{i}$ such that $\left(I\left(x_{i}, A_{i}\right) / E\left(x_{i}, A_{i}\right)\right) \geqq$ $k$. To simplify notation let $I_{i}=I\left(x_{i}, A_{i}\right)$ and $E_{i}=E\left(x_{i}, A_{i}\right)$. Without loss of generality assume that $E_{1}=\min \left\{E_{i}\right\}=1$ and $x_{1}=0$. Let $\widetilde{\mho}_{1}$ consist of all sets in $\mathfrak{F}$ which meet $A_{1}$ and $\widetilde{F}_{2}=\mathfrak{F} / \widetilde{F}_{1}$. Now divide $\widetilde{F}_{1}$ into two subfamilies $\mathscr{C}_{1}=\left\{A_{i} \in \widetilde{F}_{1}:\left\|x_{i}\right\| \leqq 1+k\right\}$ and $\mathscr{C}_{2}=\left\{A_{i} \in \mathscr{\mho}_{1}\right.$ : $\left.\left\|x_{i}\right\|>1+k\right\}$. There exists $N_{1}$ points $z_{1}, \cdots, z_{N_{1}}$ such that $(1+k) B^{n}$ is covered by the family $\left\{z_{i}+k B^{n}: i=1, \cdots, N_{1}\right\}$. For $A_{i} \in \mathscr{C}_{1}$ it follows that $x_{i} \in z_{j}+k B^{n}$ for some $j$. Since $I_{i} \geqq k E_{i} \geqq k$, it follows that $x_{i}+k B^{n} \subset A_{i}$. Moreover, $x_{i} \in z_{j}+k B^{n}$ implies that $z_{j} \in x_{i}+$ $k B^{n} \subset A_{i}$; consequently each set in $\mathscr{C}_{1}$ contains some $z_{j}$. Therefore, $\left|\mathscr{C}_{1}\right| \leqq N_{1}$.

Now let $A_{i} \in \mathscr{C}_{2}$. Since $A_{i} \cap A_{1} \neq \varnothing$ and $A_{1} \subset B^{n}$, it follows that $A_{i} \cap B^{n} \neq \varnothing$. Let $y_{i} \in\left[\mathrm{bd} B^{n}\right] \cap A_{i}$ and $w_{i}=\lambda x_{i}+(1-\lambda) y_{i}$ belong to bd $\left[(1+k) B^{n}\right]$ with $0<\lambda<1$. The set $w_{i}+\lambda\left(I_{i} B^{n}\right)$ is contained in the convex hull of the set $\left\{x_{i}+I_{i} B^{n}\right\} \cup\left\{y_{i}\right\}$ which is contained in $A_{i}$. Now $k \leqq\left\|y_{i}-w_{i}\right\|=\lambda\left\|y_{i}-x_{i}\right\| \leqq \lambda E_{i}$, which implies $\lambda I_{i} \geqq\left(k / E_{i}\right) I_{i} \geqq$ $k^{2}$. Therefore, $w_{i}+k^{2} B^{n} \subset A_{i}$. Let $u_{1}, \cdots, u_{N_{2}}$ be $N_{2}$ points such that $u_{j} \in \mathrm{bd}\left[(1+k) B^{n}\right]$ and $\mathrm{bd}\left[(1+k) B^{n}\right]$ is covered by the family $\left\{u_{j}+k^{2} B^{n}: j=1, \cdots, N_{2}\right\}$. Now $w_{i} \in u_{j}+k^{2} B^{n}$ for some $j$, which implies that $u_{j} \in w_{i}+k^{2} B^{n} \subset A_{i}$. Hence, each set in $\mathscr{C}_{2}$ contains one of the $N_{2}$ points $u_{1}, \cdots, u_{N_{2}}$; consequently $\left|\mathscr{C}_{2}\right| \leqq N_{2}$. Therefore, $\left|\mathfrak{F}_{1}\right| \leqq$ $N_{1}+N_{2}$.

To complete the proof note that since each set in $\mho_{2}$ fails to intersect $A_{1}$, it follows that either $\mho_{2}$ has the $(p-1, q, k)$-property or $\widetilde{\mho}_{2}$ fails to contain $p-1$ sets. The theorem now follows by induction on $p-q$.

For $k>0$ the proof of Theorem 2.1 can be used to obtain upper bounds for the values of $P_{n}(p, q, k)$. In the case of $k=1$, Hadwiger and Debrunner's work with families of mutually parallel parallelotopes [3, p. 32] can be used to obtain upper bounds for $P_{n}(p, q, 1)$ and if the supplementary condition $2 \leqq q \leqq p \leqq 2 q-2$ is satisfied, then $P_{n}(p, q, 1)=p-q+1$.

As a function of $k \in[0,1]$ to the positive integers union $\infty$, $P_{n}(p, q, k)$ is a decreasing function and Theorem 2.1 implies that it is 
integral-valued for $k \in(0,1]$. The following theorem implies that it is continuous from the right at each $k \in[0,1)$.

Theorem 2.2. Let $k_{0} \in[0,1)$ then $P_{n}(p, q, k) \rightarrow P_{n}\left(p, q, k_{0}\right)$ as $k \rightarrow k_{0}^{+}$.

Proof. By Lemma 1.1 it is enough to show that $|\mathfrak{F}| \leqq$ $\sup \left\{P_{n}(p, q, k): k>k_{0}\right\}$ for each finite family $\widetilde{F}$ with the $\left(p, q, k_{0}\right)$ property in $L^{n}$. For such an $\widetilde{₹}$ and $\varepsilon>0$, let $\widetilde{\mho}_{\varepsilon}=\left\{A+\varepsilon B^{n}: A \in \mathfrak{F}\right\}$. Then $\widetilde{\mho}_{\varepsilon}$ has the $\left(p, q, k_{\varepsilon}\right)$-property in $L^{n}$, where $k_{\varepsilon}>k_{0}$ and $k_{\varepsilon} \rightarrow k_{0}$ for $\varepsilon \rightarrow 0$; also, by simple compactness arguments $|\mathfrak{\mho}| \leqq \lim \inf _{\varepsilon \rightarrow 0}\left|\mathfrak{F}_{\varepsilon}\right| \leqq$ $\sup \left\{P_{n}(p, q, k): k>k_{0}\right\}$.

For $p \geqq q \geqq 2$ and $n \geqq 1$, let $D(p, q ; n)$ denote the set of points in $[0,1]$ where as a function of $k P_{n}(p, q, k)$ is discontinuous from the left. If $p, q$, and $n$ satisfy the inequalities in Corollary 1.4, then clearly $D(p, q ; n)=\varnothing$. It is unknown whether or not there exist values of $p, q$, and $n$ such that $D(p, q ; n)$ is a nonempty finite set. If $D(p, q ; n) \neq \varnothing$ then $D(p, q ; n)$ can be written in the form $\left\{\xi_{i}(p, q ; n): i \in J\right\}$, where $J$ is either the set of positive integers or an initial segment of the positive integers, with $\xi_{i+1}(p, q ; n)<\xi_{i}(p, q ; n)$. In the next section $\xi_{1}(p, q ; n)$ will be determined for certain values of $p, q$, and $n$.

3. Some examples, From Corollary 1.4 and the fact that $P_{n}(p, q, 0)=\infty$ for $n \geqq q \geqq 2$ it follows that the case with the smallest values for $p, q$, and $n$, in which the question is unanswered as to whether or not $P_{n}(p, q, 0)$ is finite or not is that of $p=4, q=3$, and $n=2$. However, it is known that $P_{2}(4,3,1)=2$. An example will now be constructed to show that $P_{2}(4,3, k) \geqq 3$ and $P_{2}(2,2, k) \geqq$ 3 for all $k$ with $0 \leqq k<1$.

ExAmple 3.1. For $m \geqq 3$ define points in $L^{2}$ as follows:

$$
\begin{gathered}
A_{m}=(-1 / 2 m, 0), \quad B_{m}=(1 / 2 m, 0), \quad C_{m}=(0,-1 / m), \quad D=(1,0), \\
E=(-1,0), \quad F_{m}=(1,-1 / m), \quad G=(-1,-1), \quad H=(1,-1), \\
I=(0,-1), \quad J=(-1,2), \quad K=(1,2), \quad L_{m}=(-1,-1+1 / 2 m) .
\end{gathered}
$$

Also, let $M_{m}$ and $N_{m}$ denote the points of intersection of the line through $C_{m}$ and $D$ with the line $x=-1 / 2 m$ and $x=1 / 2 m$, respectively, $P_{m}$ the intersection of the line through $I$ and $N_{m}$ with the line $y=-1+1 / 2 m, R_{m}$ the intersection of the line through $I$ and $M_{m}$ with the line through $E$ and $C_{m}$.

Using the above points, define sets in $L^{2}$ as follows:

$$
\begin{array}{ll}
S_{m}^{1}=\operatorname{conv}\left\{J, K, D, C_{m}, E\right\}, & S_{m}^{2}=\operatorname{conv}\left\{N_{m}, D, H, I\right\}, \\
S_{m}^{3}=\operatorname{conv}\left\{C_{m}, F_{m}, I, H\right\}, & S_{m}^{4}=\operatorname{conv}\left\{E, R_{m}, I, G\right\},
\end{array}
$$




$$
S_{m}^{5}=\operatorname{conv}\left\{E, M_{m}, P_{m}, L_{m}\right\} \text {, and } S_{m}^{6}=\operatorname{conv}\left\{A_{m}, B_{m}, N_{m}, M_{m}\right\} \text {. }
$$

Let $\widetilde{F}_{m}=\left\{S_{m}^{i}: i=1, \cdots, 6\right\}$ for $m=3, \cdots$. Figure 1 illustrates the family $\mathfrak{\mho}_{4}$. For each $m$ with $m \geqq 3$, the family $\mathfrak{\mho}_{m}$ has both the $(2,2)$-property and the $(4,3)$-property. Moreover, $\left|\mathfrak{\mho}_{m}\right|=3$ for all $m \geqq 3$. Note also that given $k<1, m$ can be chosen large enough such that $\widetilde{F}_{m}$ has both the $(2,2, k)$-property and the $(4,3, k)$-property in $L^{2}$. Consequently, $P_{2}(2,2, k) \geqq 3$ and $P_{2}(4,3, k) \geqq 3$ for all $k$ with $0 \leqq k<1$. Since as a function of $n, P_{n}(p, q, k)$ is an increasing function, it follows that $P_{n}(2,2, k) \geqq 3$ and $P_{n}(4,3, k) \geqq 3$ for all $0 \leqq k<$ 1 and $n \geqq 2$. This with the fact that $P_{n}(2,2,1)=1$ and $P_{n}(4,3,1)=$ 2 implies that $\xi_{1}(2,2 ; n)=\xi_{1}(4,3 ; n)=1$ for all $n \geqq 2$. Grünbaum [1] has given an example of a family $\widetilde{f}$ consisting of 21 circular disks in $L^{2}$ with the $(2,2)$-property such that $|\mathfrak{F}|=4$. Thus, $P_{n}(2,2, \sqrt{2} / 2) \geqq$ 4 for all $n \geqq 2$.

Theorem 3.1. If $2 \leqq q \leqq n$, then $\xi_{1}(q, q ; n)=1$.

Proof. Since $P_{n}(q, q, 1)=1$ it suffices to show that if $k<1$, then $P_{n}(q, q, k) \geqq 2$. Moreover, since $P_{n}(q, q, k) \geqq P_{n}(n, n, k)$ for $q \leqq n$ the theorem will follow by showing that $P_{n}(n, n, k) \geqq 2$ for all $k<1$. Example 3.1 implies the desired result for $n=2$, so assume that $n \geqq 3$.

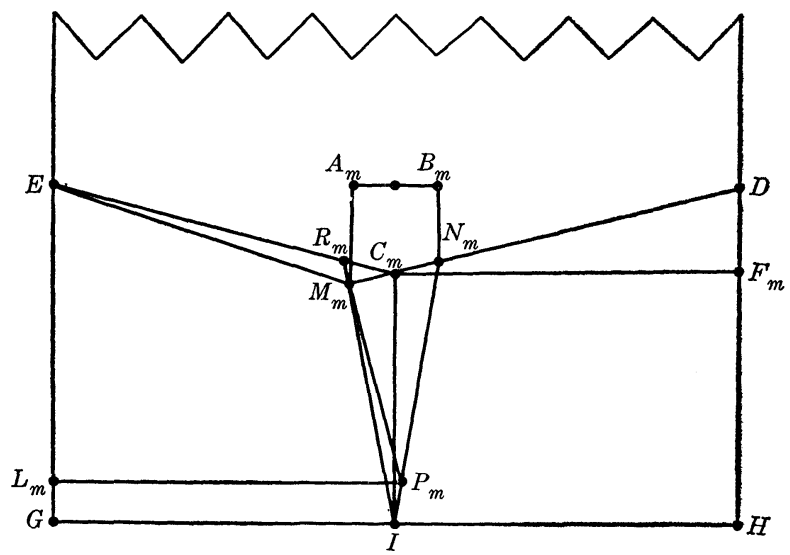

FIGURE 1

Without loss of generality, assume that $L^{n-1}$ is the subspace of $L^{n}$ which is perpendicular to the line determined by the origin and the point $(0, \cdots, 0,1)$. Since $P_{n-1}(n-1, n-1,0)=\infty$, there exists a finite family $\widetilde{F}=\left\{A_{i}: i=1, \cdots, m\right\}$ with the $(n-1, n-1,0)$-property in $L^{n-1}$ such that $|\mathfrak{\mho}| \geqq 2$. Without loss of generality, assume that $A_{i} \subset B^{n-1}$ for $i=1, \cdots, m$. Let $A_{i}^{j}$ denote the convex hull of the set $A_{i} \cup\left[B^{n}+(0, \cdots, 0,1+(1 / j))\right]$ and let $A=B^{n}+(0, \cdots, 0,-1)$. For $j=1, \cdots$ let $\mathfrak{F}_{j}=\left\{A_{i}^{j}: i=1, \cdots, m\right\} \cup\{A\}$. Then the family $\mathfrak{\mho}_{j}$ has 
the $(n, n, j /(j+1))$-property in $L^{n}$ and $\left|\widetilde{F}_{j}\right|=2$. Thus, if $0 \leqq k<1$, there exists a $j$ such that $\mathfrak{\mho}_{j}$ has the $(n, n, k)$-property in $L^{n}$ and $\left|\Im_{j}\right|=2$. Consequently, $P_{n}(n, n, k) \geqq 2$ for all $k$ with $0 \leqq k<1$.

4. Concluding remarks. The procedure used in this paper in defining the piercing function $P_{n}(p, q, k)$ depends on the set $B^{n}$. A similar development using any closed unit ball of an $n$-dimensional normed linear space could have been done. The theorems and corollaries which were proven in $\S \S 1$ through 2 would still remain true. However, the piercing function will not be identical to $P_{n}(p, q, k)$ unless the unit ball is a parallelotope. The unit ball $B^{n}$ seems to be the best of all possible choices because $P_{n}(2,2,1)=1$. This would not have been the case if the unit ball was not a parallelotope.

This paper gives rise to several unanswered questions which should be rather clear from the context. However, they could all probably be answered by answering the primary question, that is, what is the value of $P_{n}(p, q, k)$ for all $p \geqq q \geqq 2, n \geqq 2$, and $0 \leqq k \leqq 1$ ?

\section{REFERENCES}

1. B. Grünbaum, On intersections of similar sets, Portugal. Math., 18 (1959), 155-164.

2. H. Hadwiger, and H. Debrunner, Über eine variante zum hellyschen satz, Arch. der Math., 8 (1957), 309-313.

3. H. Hadwiger, H. Debrunner, and V. Klee, Combinatorial Geometry in the Plane, New York: Holt, Rinehart, and Winston, 1964.

4. E. Helly, Über mengen konvexer körper mit gemeinschaftlichen punkten, Jber. Deutsch. Math. Verein., 32 (1923).

Received September 26, 1972 and in revised form December 18, 1972. The results of this paper form part of Chapter 3 of the author's Ph. D. thesis "Intersectional Properties of Families of Compact Convex Sets", which was prepared under the guidance of Professor E. K. McLachlan at Oklahoma State University. The author wishes to express his sincere gratitude to Professor McLachlan for his helpful suggestions and criticism and also to the referee for his suggestions and comments. 


\section{PACIFIC JOURNAL OF MATHEMATICS}

\section{EDITORS}

RICHARD ARENS (Managing Editor) University of California

Los Angeles, California 90024

R. A. BeaUmont

University of Washington Seattle, Washington 98105
J. DUGUNDJI*

Department of Mathematics University of Southern California Los Angeles, California 90007

D. Gilbarg and J. Milgram Stanford University

Stanford, California 94305

\section{ASSOCIATE EDITORS}

E. F. BECKENBACH

B. H. NeUmanN

F. WOLF

K. YoSHIDA

\section{SUPPORTING INSTITUTIONS}

\section{UNIVERSITY OF BRITISH COLUMBIA CALIFORNIA INSTITUTE OF TECHNOLOGY UNIVERSITY OF CA.LIFORNIA MONTANA STATE UNIVERSITY UNIVERSITY OF NEVADA NEW MEXICO STATE UNIVERSITY OREGON STATE UNIVERSITY UNIVERSITY OF OREGON OSAKA UNIVERSITY}

UNIVERSITY OF SOUTHERN CALIFORNIA STANFORD UNIVERSITY UNIVERSITY OF TOKYO UNIVERSITY OF UTAH WASHINGTON STATE UNIVERSITY UNIVERSITY OF WASHINGTON AMERICAN MATHEMATICAL SOCIETY NAVAL WEAPONS CENTER

* C. R. DePrima California Institute of Technology, Pasadena, CA 91109, will replace J. Dugundji until August 1974. 


\section{Pacific Journal of Mathematics}

\section{Vol. 50, No. $1 \quad$ September, 1974}

Gail Atneosen, Sierpinski curves in finite 2-complexes.............. 1

Bruce Alan Barnes, Representations of $B^{*}$-algebras on Banach spaces .... 7

George Benke, On the hypergroup structure of central $\Lambda(p)$ sets ....... 19

Carlos R. Borges, Absolute extensor spaces: a correction and an

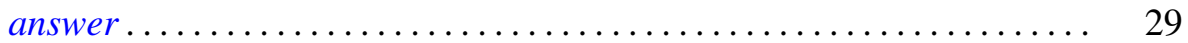

Tim G. Brook, Local limits and tripleability .................. 31

Philip Throop Church and James Timourian, Real analytic open maps .... 37

Timothy V. Fossum, The center of a simple algebra ............... 43

Richard Freiman, Homeomorphisms of long circles without periodic

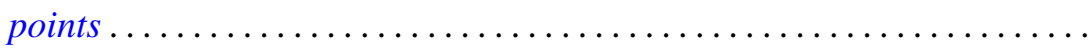

B. E. Fullbright, Intersectional properties of certain families of compact

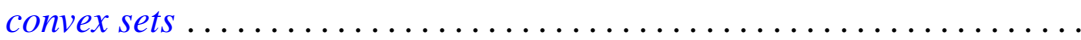

Harvey Charles Greenwald, Lipschitz spaces on the surface of the unit

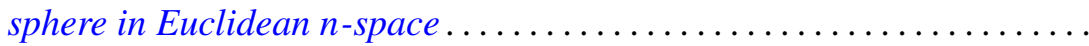

Herbert Paul Halpern, Open projections and Borel structures for

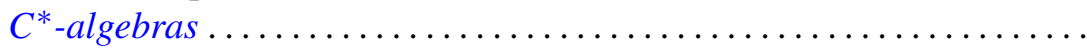

Frederic Timothy Howard, The numer of multinomial coefficients divisible

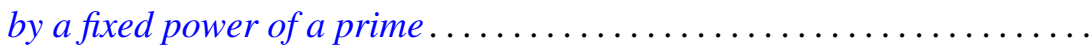

Lawrence Stanislaus Husch, Jr. and Ping-Fun Lam, Homeomorphisms of manifolds with zero-dimensional sets of nonwandering points........ 109

Joseph Edmund Kist, Two characterizations of commutative Baer rings ...

Lynn McLinden, An extension of Fenchel's duality theorem to saddle functions and dual minimax problems ................

Leo Sario and Cecilia Wang, Counterexamples in the biharmonic classification of Riemannian 2-manifolds...

Saharon Shelah, The Hanf number of omitting complete types ...

Richard Staum, The algebra of bounded continuous functions into a

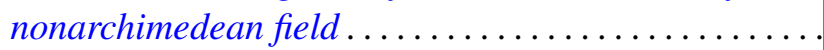

James DeWitt Stein, Some aspects of automatic continuity ..

Tommy Kay Teague, On the Engel margin

John Griggs Thompson, Nonsolvable finite groups all of whose local subgroups are solvable, $V \ldots \ldots \ldots \ldots \ldots \ldots \ldots \ldots$

Kung-Wei Yang, Isomorphisms of group extensions 